Electronic version of an article published as [European Journal of Operational Research, 2001, Vol. 131, No. 3, p. 220-228]

[DOI: http://dx.doi.org/10.1016/S0377-2217(00)00110-7] (C [Elsevier] 


\title{
Solving the generalized apportionment problem through the optimization of discrepancy functions
}

\author{
Joaquín Bautista, Ramon Companys and Albert Corominas
}

Departament d'Organització d'Empreses, Institut d'Organització i Control de Sistemes Industrials, ETSEIB, Universitat Politècnica de Catalunya, Av. Diagonal 647, 08028 Barcelona, Spain

\begin{abstract}
One of the ways to solve the classical apportionment problem (which has been studied chiefly in relation to the apportionment of seats in a chamber of representatives) is the optimization of a discrepancy function; although this approach seems very natural, it has been hardly used. In this paper, we propose a more general formalization of the problem and an optimization procedure for a broad class of discrepancy functions, study the properties of the procedure and present some examples in which it is applied.
\end{abstract}

Author Keywords: Combinatorial analysis; Apportionment problem 


\section{Introduction}

The classical apportionment problem (ApP) can be stated as follows.

A positive integer, $h$, a set, $M$, such that $|M|=m$, and nonnegative real values $q_{i}(i=1, \ldots, m)$ such that $\sum_{i=1}^{m} q_{i}=h$, which are called quotas, are given. An apportionment of $h$ is a vector $\left(x_{1}, \ldots, x_{m}\right)$ with nonnegative integer components such that $\sum_{i=1}^{m} x_{i}=h$. The objective is to find an apportionment such that the values $x_{i}$ are as close as possible to their respective quotas.

This case occurs, for example, when the elements of $M$ have associated values $p_{i} \geq 0(i=1, \ldots, m)$, with $\sum_{i=1}^{m} p_{i}=P$, and we wish to distribute $h$ proportionally to these values. Then

$q_{i}=r_{i} h$ with $r_{i}=\frac{p_{i}}{p}$

The value $h$ may correspond to the available number of indivisible units of some resource, to be distributed among the $m$ elements of $M$.

A more general problem can be defined by relaxing the conditions relative to the $q_{i}$ (i.e., nonnegativity and $\sum_{i=1}^{m} q_{i}=h$ ), although in this case they cannot properly be called quotas. We will designate this problem as the generalized apportionment problem (GApP).

The nature of the resource to be distributed and that of the elements of the set $M$ can be very diverse. The distribution of the planned units of a family of products among the specific products which it comprises, for instance, can be seen as an ApP.

The $q_{i}$ may correspond to some required or desired values to be assigned to the elements of the set $M$; if the sum of these desired values is greater than the number of available units to be distributed, $h$, we are faced with a GApP. Some examples would be the allocation of teaching staff to university departments, schools to city districts, computers to departments of a company or government body, copies of a book to libraries, servers to queuing facilities or identical machines to workers.

The ApP has been studied primarily, however, in relation to the assignment of seats in a chamber of representatives to electoral constituencies (states in the case of the United States Congress) proportionally to their population, or to political options (parties or coalitions) standing at elections proportionally to votes obtained; of course in these cases the problem is just the classical ApP.

In Section 2 of this paper we present a synthesis of the classical approaches to the ApP in its application to the apportionment of seats; in Section 3, we define procedures (which we call generalized divisor methods or GDMs) that optimize a family of general discrepancy functions, for the GApP, itself defined through very general properties, and we also establish a method for determining which discrepancy functions are optimized by any given GDM, including the classical divisor methods (DMs); in Section 4, we present some examples in which we apply the procedures given in Section 3; and finally, Section 5 includes some brief conclusions.

\section{The apportionment of seats in a chamber of representatives}

In relation to the apportionment of seats, the ApP has been dealt with most thoroughly in Balinski and Young, 1982 and Balinski and Young, 1994. Other references on the subject are given in this section. 
We present a synthesis of the various ways in which the problem has been dealt with and a brief discussion leading on to the use of discrepancy functions, which constitute the basis of the developments contained within this paper. A more complete description of the methods and their properties can be found in Balinski and Young (1982).

As regards the apportionment of seats, the first procedures to be formalized (Hamilton and Jefferson) date from the end of the 18th century.

Nowadays it seems very natural to us to consider the ApP as being an optimization problem in which the aim is to minimize a discrepancy function between $x_{i}$ and the quotas. Historically, however, the procedures adopted were based on some simple rule for obtaining $x_{i}$ from $q_{i}$ without explicitly posing any discrepancy function, even though one or more were sometimes optimized by the procedure.

In Hamilton's method, which is given many other names, one of the most frequently used of which is the largest fractions or LF method, each state is apportioned the integral part of $q_{i}$ and the remaining seats, one by one, according to the order of the fractional parts of the same $q_{i}$ (from larger to smaller). In DMs a common divisor, $\lambda$, is sought such that the quotients $p_{i} / \lambda$ rounded with a specific rule in each method (which characterizes that method), add up to $h$ ( $\lambda$ can be interpreted as an approximation to the number of inhabitants per seat, which ideally, according to the "one man, one vote" principle, should be the same for all states, although, in general, due to the integrity of the seats, this ideal cannot be achieved). For example, in Jefferson's method $x_{i}$ is the largest integer $\leq p_{i} / \lambda$ (the quotient is truncated), whereas in Webster's it is the largest integer $\leq\left(p_{i} / \lambda\right)+0.5$ (the quotient is rounded in the usual way).

The determination of $\lambda$ is not difficult but does involve a process of trial and error and, moreover, on the whole there is no single value for this parameter. In practice, DMs are applied by using iterative algorithms for the successive apportionment of seats: divisors, $d(a)$, with $a \geq 0$ and integer, are defined such that $a \leq d(a) \leq a+1$ and $d(a)<d(a+1) ; a_{i}$ being the number of seats apportioned to the state $i$ after a certain number of iterations, at each iteration a seat is awarded to one of the states to which the greatest value of the quotient $p_{i} / d\left(a_{i}\right)$ (or the quotient $q_{i} / d\left(a_{i}\right)$, given the proportionality between $p_{i}$ and $\left.q_{i}\right)$ corresponds. Similarly, the quotients $q_{i} / d(a)(0 \leq a \leq h)$ can be calculated and the seats apportioned according to the order of these quotients, from larger to smaller.

Clearly, then, DMs possess the property that solutions always exist for which $x_{i}(h+1) \geq x_{i}(h) \forall i$, or house monotonicity, property $\mathrm{H}$, which means that if the house size increases the number of seats awarded to any option does not diminish. This is not the case, for example, with the LF or Hamilton's method.

Of the infinite variety of DMs, the five that are considered as being traditional or historical are those presented and defined in Table 1 .

Table 1. Five traditional DMs

\begin{tabular}{llllll}
\hline Method & Adams & Dean & Hill & Webster & Jefferson \\
\hline$d(a)$ & $a$ & $\frac{a(a+1)}{a+1 / 2}$ & $\sqrt{a(a+1)}$ & $a+\frac{1}{2}$ & $a+1$ \\
\hline
\end{tabular}


Traditionally, once a method was defined, the properties it possessed were studied. Later on, a different approach was adopted: to postulate properties and find methods possessing them. For example, Still (1979) postulates that a method should be H and Q (Q or quota being such that $\left\lfloor q_{i}\right\rfloor \leq x_{i} \leq\left\lceil q_{i}\right\rceil\lfloor y\rfloor$ being the integral part of $y$ and $\left\lfloor q_{i}\right\rfloor=-\left\lceil-q_{i}\right\rceil$ ), and constructs a family of methods with these properties.

Huntington (1928) was the first to introduce the concept of optimization in the approach to the ApP. Once an inequality measurement had been defined between two states, the aim was to find a locally optimal apportionment of seats, i.e., one in which no transfer of a seat from one state to another would improve the inequality measurement for the pair of states concerned. Naturally, the method used to find the solution depends on how the inequality measurement is defined; it is notable that those used by Huntington lead precisely to the five traditional DMs in Table 1, all of which were already known at that time.

The optimization of a general discrepancy function has never, then, been a starting point for a definition of the typically applied methods. Nevertheless, in Balinski and Young (1983) several functions are suggested to measure the "error" of an apportionment. In Athanasopoulos (1993), the possibility of optimizing a function is mentioned and several functions are suggested too. In Ernst (1994), the ability of some methods to optimize certain functions is one of the arguments used in the very interesting legal debate that is set forth. As a rule, the fact of a method optimizing a discrepancy function appears as a property; thus, for example, the following has been proved.

Proposition 1. Hamilton minimizes $\sum_{i=1}^{m} f\left(x_{i}-q_{i}\right)$, with $f$ convex and such that $f(0)=0$ (Bautista et al., 1994).

Proposition 2. Webster minimizes $\sum_{i=1}^{m}\left(x_{i}-q_{i}\right)^{2} / q_{i}$ (Lucas, 1978, p. 379; Balinski and Young, 1982, p. 105; the proof is based on an exchange argument).

Proposition 3. Hill minimizes $\sum_{i=1}^{m}\left(x_{i}-q_{i}\right)^{2} / x_{i}$ (Lucas, 1978, p. 379).

It is not our intention to discuss the validity of the procedures adopted to date for the apportionment of seats, but they need not be the most appropriate for other circumstances in which the ApP may present itself. In some cases, it may be very natural to try to minimize a given discrepancy function yet have no reason to impose properties such as $\mathrm{H}$, which is considered unavoidable when apportioning seats. In such cases, there is a need for some procedure which will optimize the discrepancy function adopted.

It would also seem to be desirable to be able to determine easily which types of discrepancy function a given procedure optimizes, since, at the very least, this helps us to understand what the use of the method implies. 


\section{Generalized divisor methods (GDMs)}

The problem posed is as follows:

Given: $m, h$ (positive integers); real values $q_{i}(i=1, \ldots, m)$; and functions $f_{i}\left(q_{i}, x_{i}\right)(i=1, \ldots, m)$, defined for nonnegative integer values of $x_{i}$ and such that

$f_{i}\left(q_{i}, x_{i}\right) \leq \frac{1}{2}\left[f_{i}\left(q_{i}, x_{i}-1\right)+f_{i}\left(q_{i}, x_{i}+1\right)\right]_{(1)}$

(these $f_{i}$ are functions of a single integer variable, $x_{i}$, since $q_{i}$ intervenes as a parameter). To solve:

PR1

$[\mathrm{MIN}] z_{s}=\sum_{i=1}^{m} f_{i}\left(q_{i}, x_{i}\right)$

$\sum_{i=1}^{m} x_{i}=h$

$x_{i} \geq 0$ and integer.

It is clear that for (1) to be fulfilled it is sufficient for $f_{i}$ to be convex, but the convexity, which is a stronger property, is not a necessary condition (recall that the functions must be defined for the integer values of the variables, but need not be defined for fractional values of them, as can happen in some applications; for instance, the functions may be defined by means of a table).

Of course one can devise "reasonable" functions not fulfilling property (1). For instance, if we consider the functions, or classes of functions, suggested in Balinski and Young (1983), the class of objective functions dealt with in this paper includes class (1) in Balinski and Young but not their function (2).

The functions $f_{i}$ may be of the same type for all values of $i$ (i.e., $f_{i}\left(q_{i}, x_{i}\right)=f\left(q_{i}, x_{i}\right) \forall i$ ), but this is not necessarily the case. If the $q_{i}$ are the desired values for the variables $x_{i}$ and the $f_{i}$ are defined for the real numbers these can possess also the property

(5)

$f_{i}\left(q_{i}, q_{i}\right)=0 \quad \forall i$.

However, initially we only suppose property (1) to be fulfilled.

The mathematical program PR1 can be solved by dynamic programming (being very similar to the knapsack problem). Now, we can write

$f_{i}\left(q_{i}, x_{i}\right)=\sum_{k=1}^{x_{i}}\left[f_{i}\left(q_{i}, k\right)-f_{i}\left(q_{i}, k-1\right)\right]+f_{i}\left(q_{i}, 0\right)=f_{i}\left(q_{i}, 0\right)+\sum_{k=1}^{x_{i}} \delta_{i k}$

with

$\delta_{i k}=f_{i}\left(q_{i}, k\right)-f_{i}\left(q_{i}, k-1\right)$. 
Then

$\delta_{i k} \leq \delta_{i, k+1}, \quad(7)$

since

$\left[\delta_{i k} \leq \delta_{i, k+1}\right] \Leftrightarrow\left[f_{i}\left(q_{i}, k\right)-f_{i}\left(q_{i}, k-1\right) \leq f_{i}\left(q_{i}, k+1\right)-f_{i}\left(q_{i}, k\right)\right] \Leftrightarrow\left[f_{i}\left(q_{i}, k\right) \leq \frac{1}{2} f_{i}\left(q_{i}, k-1\right)+f_{i}\left(q_{i}, k+1\right)\right]$,

which is property (1).

Therefore, PR1 is equivalent to

PR2

$[\mathrm{MIN}] z_{s}=\sum_{i=1}^{m} \sum_{k=1}^{h} \delta_{i k} y_{i k}+\sum_{i=1}^{m} f_{i}\left(q_{i}, 0\right)$

$\sum_{i=1}^{m} \sum_{k=1}^{h} y_{i k}=h^{(9)}$

$y_{i, k+1} \leq y_{i k} \quad \forall i ; k=1, \ldots, h-1, \quad{ }^{(10)}$

$y_{i k} \in\{0,1\} \forall i, k . \quad(11)$

We shall say that a sequence of $\delta_{i k}$, for a given $h$, is a well-ordered sequence (WOS) if and only if $\left[\left\{\delta_{i k}<\delta_{i k}^{\prime}\right\} \vee\left\{\left(\delta_{i k}=\delta_{i k}^{\prime}\right) \wedge\left(i=i^{\prime}\right) \wedge\left(k<k^{\prime}\right)\right\}\right] \Rightarrow\left[\delta_{i k}\right.$ precedes $\left.\delta_{i k}^{\prime}\right]$.

In a WOS, the values of $\delta$ are nondecreasing and $\forall i k<k^{\prime}$ implies that $\delta_{i k}$ precedes $\delta_{i k}{ }^{\prime}$, since, by (7), either $\delta_{i k}<\delta_{i k}{ }^{\prime}$ or $\delta_{i k}=\delta_{i k}{ }^{\prime}$, and in the latter case the tie is resolved by placing $\delta_{i k}$ before $\delta_{i k}$. Obviously, there always exists at least one WOS (and more than one if there are ties between different $\delta$ corresponding to two different elements of $M$, that can be resolved arbitrarily).

Obviously, then, the solution which corresponds to the first $h \delta_{i k}$ in any WOS is an optimal solution of PR2 and, therefore, of PR1 (property (7) and the definition of a WOS guarantees the fulfilment of constraints (10)). Furthermore, any optimal solution of PR2 (and, therefore, of PR1) corresponds to the first $h \delta_{i k}$ in some WOS.

Therefore, we can state the following:

Theorem 1. If the functions $f_{i}\left(q_{i}, x_{i}\right)$ possess property (1), then a solution is optimal for the function $z_{S}$ if and only if it corresponds to the first helements of a WOS of the $\delta_{i k}$. And also the following:

Corollary 1. Given values of $\delta_{i k}$ which fulfil condition (7), the solution determined by the first $h$ values of a WOS optimizes the discrepancy function $z_{S}=\sum_{i=1}^{m} f_{i}\left(q_{i}, x_{i}\right)$ with $f_{i}\left(q_{i}, x_{i}\right)=\sum_{k=1}^{x}{ }_{i} \delta_{i k}+f_{i}\left(q_{i}, 0\right)$. Here $f_{i}\left(q_{i}, 0\right)$ is an arbitrary constant which, if so wished, can be determined by imposing a condition such as (5). In such a case,

$f_{i}\left(q_{i}, 0\right)=-\phi_{i}\left(q_{i}\right)$,

with $\phi_{i}$ defined for the real numbers and such that

$\phi_{i}(x)=\sum_{k=1}^{x} \delta_{i k} \forall x \in \mathbb{N}$ 
We can also take $f_{i}\left(q_{i}, 0\right)=-\delta_{i 1}$, instead of $f_{i}\left(q_{i}, 0\right)=-\phi_{i}\left(q_{i}\right)$, and then $f_{i}\left(q_{i}, 1\right)=0$ and $f_{i}\left(q_{i}, x_{i}\right)=\sum_{k=2}{ }_{i}^{x} \delta_{i k}$ $\forall x_{i} \geq 2$.

Moreover, the following is immediate:

Corollary 2. An optimal solution for $z_{S}=\sum_{i=1}^{m} f_{i}\left(q_{i}, x_{i}\right)$ is also optimal for $Z_{S}=\sum_{i=1}^{m} F_{i}\left(q_{i}, x_{i}\right)$, with $F_{i}$ such that $F_{i}\left(q_{i}, k\right)-F_{i}\left(q_{i}, k-1\right)=g\left(\delta_{i k}\right)$, where $g$ is a monotonic nondecreasing function (we will use the notation $\left.\Delta_{i k}=F_{i}\left(q_{i}, k\right)-F_{i}\left(q_{i}, k-1\right)\right)$.

The determination of the optimal solution does not require the calculation of all the $\delta_{i k}$; it is only necessary to determine the first $h$ elements of a WOS, which can be done iteratively by using the following algorithm:

GDMA:

$x_{i}=0 \quad \forall i ; \quad \theta_{i}=\delta_{i 1} \quad \forall i$.

Repeat $h$ times :

$$
\begin{aligned}
& \text { Find } i^{*} \mid \theta_{i}=\min _{i} \theta_{i} ; \\
& x_{i^{*}}=x_{i^{*}}+1 ; \theta_{i^{*}}=\delta_{i^{*}, x_{i^{*}}+1} .
\end{aligned}
$$

If there are ties, when finding $i^{*}$, these can be resolved arbitrarily. The execution of the algorithm implies $2 m+h$ evaluations of the functions $f_{i}$ (since $\delta_{i k}=f_{i}\left(q_{i}, k\right)-f_{i}\left(q_{i}, k-1\right)$ ) and $m \cdot h$ comparisons.

Let $Z_{M}\left(x_{1}, \ldots, x_{m}\right)=\max _{i \mid x i>0} \delta_{i, x i}$. The value of this function for a WOS, $Z_{M}{ }^{*}$, coincides with that of the $\delta$ occupying the position $h$; the solution defined for this sequence minimizes $Z_{M}$ because if there were a solution $\bar{x}_{i}(i=1, \ldots, m)$ such that $\delta_{i, x_{i}}<Z_{M}^{*} \forall i \mid \overline{x_{i}}>0$, there would then be at least $h \delta_{i k}$ strictly smaller than $Z_{M}{ }^{*}$, contradicting the supposition that the sequence is well ordered. An analogous reasoning shows that the sequence defined by a WOS maximizes the function $z_{M}=\min _{i} \delta_{i, x i+1}$ (the value of which for this solution coincides with that of the $\delta$ occupying the position $h+1$ in the sequence). Therefore, we can state the following two corollaries:

Corollary 3. An optimal solution for $z_{S}$ also minimizes $Z_{M}=\max _{i \mid x i>0} \delta_{i, x i}$.

Corollary 4. An optimal solution for $z_{S}$ maximizes $z_{M}=\min _{i} \delta_{i, x i+1}$ and therefore, if all the $\delta$ have the same sign, minimizes

$$
\frac{1}{z_{M}}=\frac{1}{\min _{i} \delta_{i, x_{i}+1}}=\max _{i} \frac{1}{\delta_{i, x_{i}+1}} .
$$

The term GDMs is justified inasmuch as DMs can be regarded as a particular case of them. Indeed, in the latter the units are allocated following the nonincreasing order of the quotients $q_{i} / d(k-1)$, and in the GDM the nondiminishing order of the $\delta_{i k}$; then if we define the $\delta_{i k}$ as follows:

$\delta_{i k}=\frac{d(k-1)}{q_{i}}$. 
the nondiminishing order of the $\delta_{i k}$ is a nonincreasing order of the $q_{i} / d(k-1)$.

For example, for Jefferson's method $\delta_{i, x i}=x_{i} / q_{i}$ and for Adams's $\delta_{i, x i+1}=x_{i} / q_{i}$.

Consequently, the following well-known results (Balinski and Young, 1982, p. 105) are immediately obtained from Corollaries 4 and 3, respectively,

Adams minimizes $\max _{i} \frac{q_{i}}{x_{i}}$.

Jefferson minimizes $\max _{i} \frac{x_{i}}{q_{i}}$.

(by Corollary 3 , Jefferson minimizes $\max _{i \mid x i>0}\left(x_{i} / q_{i}\right)$, which is equal to $\max _{i}\left(x_{i} / q_{i}\right)$ ).

If the values of $x_{i}$ are bounded, the necessary modification of the optimization procedure of the discrepancy function is immediate; in particular, in the ApP the property quota, Q (the definition of which was given in Section 2), or the properties lower quota, LQ, $\left(x_{i} \geq\left\lfloor q_{i}\right\rfloor \forall i\right)$ or upper quota, UQ $\left(x_{i} \leq\left\lceil q_{i}\right\rceil \forall i\right)$, can be imposed on the solution.

When there are boundary constraints, then on the whole the optimal solution does not coincide with the constrained one, but for each of these properties functions exist for which the nonconstrained optimal solution possesses the property (or for which an optimal solution possessing the property always exists).

For example, let us consider the property Q. A sufficient condition for the existence of an optimal solution which is $\mathrm{Q}$ is

$\delta_{i\left\lceil q_{i}\right\rceil} \geq \delta_{j\left\lfloor q_{j}\right\rfloor} \forall i$, j and $\delta_{i\left\lceil q_{i}\right\rceil+1} \geq \delta_{j\left\lceil q_{j}\right\rceil} \forall i, j, \quad(13)$

since a WOS then exists in which the units corresponding to $x_{i} \leq\left\lfloor q_{i}\right\rfloor$ precede those corresponding to $\left\lfloor q_{i}\right\rfloor<x_{i} \leq\left\lceil q_{i}\right\rceil$ and the latter precede those for which $x_{i}>\left\lceil q_{i}\right\rceil$. Condition (13) is fulfilled, for example, for $f_{i}\left(q_{i}, x_{i}\right)=\left|x_{i}-q_{i}\right|^{c} \quad(c \geq 1)$ and for any function $f_{i}\left(q_{i}, x_{i}\right)=f\left(x_{i}-q_{i}\right)$ which is convex, nonnegative and such that $f(0)=0$. It is easy to see that the GDM procedure coincides for these functions with Hamilton's or the LF procedure (an alternative proof of this result can be found in Bautista et al., 1994).

Let us now suppose that the functions $f_{i}$ have a diminishing branch (to the left of $q_{i}$ ) and an increasing branch:

$\delta_{i k}<0 \quad \forall k \leq\left\lfloor q_{i}\right\rfloor$ and $\delta_{i k}>0 \quad \forall k \geq\left\lceil q_{i}\right\rceil+1$.

Consequently, in any WOS all units violating property UQ are located after those which are sufficient to satisfy LQ, and therefore, if an optimal solution violates UQ it satisfies LQ. We can then state the following:

Theorem 2. If the functions $f_{i}$ possess properties (1) and (14), the solutions which minimize $z_{S}$ possess property $L Q$ or property $U Q$. 
Let us now consider $h$ as a parameter. In order for the procedure to be $\mathrm{H}$ it is sufficient for the order of the $\delta_{i k}(h)$ not to depend on $h$; for this to be the case, it is sufficient for them to be able to be written as

$\delta_{i k}(h)=\rho(h) \gamma(i, k)+C$,

where $\rho(h)$ is a function invariant in sign, $\gamma(i, k)$ constants depending on $i$ and $k$ and $C$ is a constant. This occurs, for example, with $q_{i}(h)=r_{i} h \forall i$ and

$$
f_{i}\left(q_{i}(h), x_{i}\right)=\frac{\left[x_{i}-q_{i}(h)\right]^{2}}{q_{i}(h)}
$$

(since it gives

$$
\delta_{i k}(h)=\frac{2 k-1-2 q_{i}(h)}{q_{i}(h)}=\frac{1}{h} \frac{2 k-1}{r_{i}}-2 ;
$$

the procedure coincides in this case with Webster's - recall Proposition 2), but not with $f_{i}\left(q_{i}(h), x_{i}\right)=\left|x_{i}-q_{i}(h)\right|^{c}(c \geq 1)$.

\section{Examples}

Theorem 1 and the algorithm inferred from it (GDMA) allow us to optimize the function $z_{S}$ obtained by summing several $f_{i}$ possessing property (1), and also to ascertain whether the procedure coincides with some more specific procedure which is already known, while Corollaries 1 and 2, given a procedure belonging to the GDM class, allow us to determine functions $f_{i}$ for which it optimizes $z_{S}$.

At this point, we shall illustrate these possibilities with some examples.

\subsection{Optimization of given functions}

Once it has been ascertained that the functions $f_{i}$ possess property (1), all that remains is to calculate the $\delta$ and form a WOS (or apply GDMA).

For example, for

$$
f_{i}\left(q_{i}, x_{i}\right)=\frac{\left(x_{i}-q_{i}\right)^{2}}{x_{i}}, \delta_{i k}=\frac{\left(k-q_{i}\right)^{2}}{k}-\frac{\left(k-1-q_{i}\right)^{2}}{k-1}=1-\frac{q_{i}^{2}}{k(k-1)}
$$

and therefore, a sequence in nondiminishing order of the $\delta$ corresponds to a nonincreasing order of the quotients $q_{i}^{2} / k(k-1)$ or of the quotients $q_{i} / \sqrt{k(k-1)}$ which amounts to the same (i.e., the method coincides in this case with Hill's; recall Proposition 3).

As a second example, if we consider the optimization of $\sum_{i=1}^{m}\left(\left(x_{i}-q_{i}\right) / q_{i}\right)^{2}$ we reach

$$
\delta_{i k}=\frac{2 k-1}{q_{i}^{2}}-\frac{2}{q_{i}} .
$$

We have used this objective function and GDMA to calculate the number of workers to be assigned to working centers in a service system. 
In another application (Bautista et al., 1996) we have used the function $\sum_{i=1}{ }^{n} w_{i}\left(q_{i}-x_{i}\right)^{2}$ to apportion $h$ units of a family of products among the specific products belonging to it.

\subsection{Functions $f_{i}$ for which a procedure minimizes $z_{S}$}

We shall study a number of methods, including the five traditional DMs.

Firstly, let us take DMs with $d(a)=a+\alpha$; this family of DMs includes Adams's $(\alpha=0)$, Webster's $(\alpha=0.5)$ and Jefferson's $(\alpha=1)$.

From

$\delta_{i k}=\frac{d(k-1)}{q_{i}}=\frac{k+\alpha-1}{q_{i}}$

we get

$f\left(q_{i}, x_{i}\right)=\sum_{k=1}^{x_{i}} \delta_{i k}=\frac{1}{2 q_{i}}\left[x_{i}^{2}+(2 \alpha-1) x_{i}\right]$

and minimizing $\sum_{i=1}^{m} f\left(q_{i}, x_{i}\right)$ is equivalent to minimizing

$\sum_{i=1}^{m} \frac{\left[x_{i}-\left(q_{i}+0.5-\alpha\right)\right]^{2}}{q_{i}}$

(recall the condition $\sum_{i=1}^{m} x_{i}=h$ ).

In Hill's procedure, $\delta_{i k}=\sqrt{k(k-1)} / q_{i}$; therefore, we immediately obtain

$f\left(q_{i}, x_{i}\right)=\frac{1}{q_{i}} \sum_{k=1}^{x_{i}} \sqrt{k(k-1)}$.

Now, we can use Corollary 2, and with

$\Delta_{i k}=-\frac{1}{\delta_{i k}^{2}}(k=2, \ldots, m)$ and $\Delta_{i 1} \leq \Delta_{j 2} \forall i, j$.

we get either

$z_{S}=\sum_{i=1}^{m} \frac{q_{i}^{2}}{x_{i}}$

or, given that $\sum_{i=1}^{m} x_{i}=h$,

$z_{S}=\sum_{i=1}^{m} \frac{\left(x_{i}-q_{i}\right)^{2}}{x_{i}}$

However, if we do $\Delta_{i k}=\delta_{i k}^{2}$, we get

$f\left(q_{i}, x_{i}\right)=\frac{1}{3 q_{i}^{2}}\left(x_{i}-1\right) x_{i}\left(x_{i}+1\right)$ and $z_{S}=\sum_{i=1}^{m} \frac{x_{i}^{3}-q_{i}^{3}-\left(x_{i}-q_{i}\right)}{q_{i}^{2}}$ 
and for $\Delta_{i k}=\ln \delta_{i k}^{2}(k=2, \ldots, m)$ and $\Delta_{i 1} \leq \Delta_{j 2} \forall i, j$ :

$f\left(q_{i}, x_{i}\right)=\ln \frac{\left(x_{i} !\right)^{2}}{x_{i} q_{i}^{2\left(x_{i}-1\right)}}$

and

$z_{S}=\ln \prod_{i=1}^{m} \frac{\left(x_{i} !\right)^{2}}{x_{i} q_{i}^{2\left(x_{i}-1\right)}}$ or $\quad z_{S}=\ln \prod_{i=1}^{m} \frac{\left(x_{i} !\right)^{2}}{x_{i} q_{i}^{2\left(x_{i}-q_{i}\right)-1}\left(\Gamma\left(q_{i}+1\right)\right)^{2}}$.

where $\Gamma$ is the Euler gamma function.

Finally, let us consider Dean's procedure:

$d(k)=\frac{k(k+1)}{k+1 / 2} ; \delta_{i k}=\frac{d(k-1)}{q_{i}}=\frac{2 k(k-1)}{(2 k-1) q_{i}} ;$

if we do

$\Delta_{i k}=\ln \frac{\delta_{i k}}{2}(k=2, \ldots, m)$ and $\Delta_{i 1} \leq \Delta_{j 2} \forall i, j$.

we get

$f_{i}\left(q_{i}, x_{i}\right)=\ln \frac{\left(x_{i} !\right)^{3} 2^{x_{i}-1}}{x_{i}^{2} q_{i}^{x_{i}-1}\left(2 x_{i}-1\right) !}$

and

$z_{S}=\ln \prod_{i=1}^{m} \frac{\left(x_{i} !\right)^{3} 2^{x_{i}-1}}{x_{i}^{2} q_{i}^{x_{i}-1}\left(2 x_{i}-1\right) !}$ or $\quad z_{S}=\ln \prod_{i=1}^{m} \frac{\left(x_{i} !\right)^{3} 2^{x_{i}-q_{i}} \Gamma\left(2 q_{i}\right)}{x_{i}^{2} q_{i}^{x_{i}-q_{i}-2}\left(2 x_{i}-1\right) !\left(\Gamma\left(q_{i}+1\right)\right)^{3}}$.

Corollaries 3 and 4 can easily be applied to all these procedures.

\section{Conclusions}

The ApP is a classical problem with numerous and varied applications (the typical one being the apportionment of seats in a chamber of representatives). One way to approach it is the optimization of a discrepancy function.

We have presented a more general formalization of the problem and we have proposed an optimization procedure for a very broad class of discrepancy functions; this procedure can be regarded as a generalization of the DMs, which have been developed in relation to the apportionment of seats, and also includes, as specific cases, other procedures for the apportionment of seats which do not belong to the DM group. We have also presented the properties of the procedure and, lastly, some examples of optimization of specific functions and of the determination of families of functions which are optimized by procedures that are a particular case of the general procedure proposed.

One possible line of research is to extend or adapt the approach presented in this paper to the resolution of minmax problems. 


\section{Acknowledgements}

This research has been supported by the CICYT (grant TAP95-0420).

\section{References}

Athanasopoulos, B.D., 1993. The apportionment problem and its application in determining political representation. $\Sigma \Pi O Y \triangle A I / S P O U D A I 433-4$, pp. 212-237.

Balinski, M.L., Young, H.P., 1982. Fair Representation. Yale University Press, New Haven, CT.

Balinski, M.L. and Young, H.P., 1983. Apportioning the United States House of Representatives. Interfaces 13 4, pp. 35-43.

Balinski, M.L., Young, H.P., 1994. Apportionment. In: Pollock, S.M.= Rothkopf, M.H., Barnett, A. (Eds.), Operations Research and the Public Sector, Handbooks in Operations Research and Management Science, vol. 6. North-Holland, Amsterdam.

Bautista, J., Companys, R., Corominas, A., 1994. Modeling and solving the production rate variation (PRV) problem. DIT 94/19, DOE, ETSEIB-UPC.

Bautista, J., Companys, R. and Corominas, A., 1996. Artemisa: Un sistema de ayuda a la programación en una empresa del sector del automóvil. Dirección y Organización 16, pp. $34-42$.

Ernst, L.R., 1994. Apportionment methods for the House of Representatives and the court challenges. Management Science 40 10, pp. 1207-1227.

Huntington, E.V., 1928. The apportionment of representatives in Congress. Transactions of the American Mathematical Society 30, pp. 85-110.

Lucas, W.F., 1978. The apportionment problem. In: Brams, S.J., Lucas, W.F., Straffin Jr., P.D. (Eds.), Political and Related Models. Springer, Berlin, pp. 358-396.

Still, J.W., 1979. A class of new methods for congressional apportionment. SIAM Journal of Applied Mathematics 37 2, pp. 401-418. 